Las consecuencias demográficas de la austeridad en América Latina: aspectos metodológicos

\title{
Ralph Hakkert*
}

Se analiza el impacto demográfico que tuvo la crisis económica de los años ochenta en el Tercer Mundo, con especial atención en América Latina. El estudio pone el énfasis en los problemas metodológicos involucrados en la medición de dichos impactos. Aparte de las siempre presentes dificultades para obtener información actual sobre la fecundidad, la mortalidad y especialmente la migración en el contexto de que las fuentes de datos son deficientes y escasas, se discuten los problemas conceptuales inherentes al establecimiento de las relaciones. Éstos incluyen la dificultad de.diferenciar entre efectos estructurales y efectos a corto plazo, especialmente en fecundidad; la presencia de desfases y estructuras de tiempos diferentes; la necesidad de distinguir entre tipos de oscilaciones económicas y los efectos para contrarrestar las que pueden ocultar las interacciones que se esperan, en especial en el caso de la mortalidad. La migración, quizá el componente demográfico más sensitivo a este respecto, es también un punto en donde la dirección esperada del impacto es más dificil de establecer. Estón en discusión las diferencias entre los análisis de corte transversal comunes en la literatura demográfica corriente y la visión longitudinal que se necesita para llevar a cabo estudios de esta clase.

\section{Introducción}

El 28 de mayo de 1984 uno de los grandes periódicos de San Pablo describió las repercusiones de la política económica de recesión del FMI en la población de Brasil de la siguiente manera:

Diecisiete meses después del acuerdo en el Fondo Monetario Internacional, Brasil entra en su cuarto año de recesión caracterizado por hambre, desempleo, aumento de la delincuencia y un número creciente de favelas. Los números son irrefutables y alarmantes: uno de cada seis trabajadores está desempleado, diez millones de brasileños reciben la mitad del salario mínimo legal; el hambre es ya una de las diez causas más frecuentes de mortalidad infantil en el Estado de San Pablo, crece el saqueo de comida y contenedores de combustible, aumentan los centros de salud de la zona metropolitana de San Pablo. Llega al $10 \%$ el índice de mortalidad infantil en las favelas de

* Centro de Planeación Regional y Urbanización (CEDEPLAR), Universidad Federal de Minas Gerais, Belo Horizonte, Brasil. 
Belo Horizonte [casi la misma proporción en la zona depauperada del Noreste). (Folha de São Paulo, 28 de mayo de 1984.)

Es muy intuitiva la idea de que los ajustes estructurales que se hicieron en América Latina y otros países menos desarrollados durante el decenio de 1980 influyeron en las tendencias demográficas actuales.

Las razones son bastante obvias. Así pues, la política ortodoxa tuvo gran efecto recesivo en las economías. En consecuencia, el desempleo aumentó y los ingresos bajaron, por lo que grandes grupos de la población quedaron por debajo del mínimo de subsistencia. Desde 1980 hasta 1984, el promedio de desempleo urbano en 12 países de América Latina aumentó de 5.8 a $7.9 \%$ [Klein y Wurgaft, 1985). ${ }^{1}$ Chile tuvo los niveles más altos, de $11.7 \%$ aumentó a $18.5 \%$. En Bolivia, Uruguay y Venezuela los porcentajes se duplicaron, en alrededor de 7 hasta 13-15\% [García y Tokman, 1985). Aunque el desempleo disminuyó algo después de 1985 en Brasil, Chile y Venezuela, no volvió a los niveles que tenía antes de 1980. El sector informal que se expandió rápidamente en 10 países disminuyó sólo un poco en Chile y Uruguay; en Brasil y Costa Rica, su crecimiento superó con creces al del sector formal (Banco Mundial, 1986, PREALC, 1985b). Los ingresos en este sector disminuyeron a medida que aumentaba su tamaño [García y Tokman, 1985). En Costa Rica, Brasil, Argentina y Perú, disminuyeron hasta 23.5-39.3 por ciento.

El problema no se limita al sector informal. Entre 1980 y 1985 , el ingreso per cápita promedio en los 23 países de América Latina cayó $9 \%$ (UNICEF, 1987). Una excepción, al menos durante 1983, fue Colombia (García y Tokman, 1985). En Costa Rica, los salarios reales cayeron casi $40 \%$ durante los años previos al ajuste (19791982), pero aumentaron $26.4 \%$ durante el periodo de ajuste en 1983-1984 (MTSS, 1985). Mientras tanto, el número de jefes de familia que ganaban el mínimo aumentó de $24.4 \%$ en 1979 a $30.8 \%$ en 1982 (PREALC, 1985a). Durante la primera mitad de la década, los salarios reales bajaron $16 \%$ en Chile, casi $30 \%$ en México, y $18 \%$ en San Pablo (UNICEF, 1987). En 1983, los reajustes del salario mijnimo por debajo de la inflación causaron $20 \%$ de pérdida en el ingreso real de los brasileños (Singer, 1985). Aunque los efectos de la recesión en el desempleo que comenzó en 1987 no han sido hasta ahora tan devastadores como en 1982-1983, si han tenido severas consecuencias.

\footnotetext{
${ }^{1}$ Los 12 paises considerados son: Argentina, Bolivia, Brasil, Chile, Colombi Costa Rica, México, Panamá, Paraguay, Perú, Uruguay y Venezuela.
} 
Para hacer frente a la situación, los obreros trabajan más horas, buscan otro empleo y trabajo pare sus esposas e hijos. Además, las familias han cambiado sus normas de consumo. En Costa Rica, donde la pobreza aumentó entre 1979 y 1982, disminuyó el consumo de carne y leche (Céspedes, 1985). El consumo de carne de res per cápita en Brasil cayó de $22 \mathrm{~kg}$ por año en 1977 a 15 en 1985 y 13 en 1987. A peșar de la falta de datos cuantitativos, la prensa brasileña informó de manera sistemática que las familias de clase trabajadora dejaban sus casas y se mudaban a las favelas (Nitrini, 1981).

Además de los efectos recesivos, la polftica económica provocó la disminución general de gasto social, especialmente en salud y educación. En América Latina, los gastos reales per cápita en estos rubros bajaron cerca de 60\% entre 1979 y 1983 (UNICEF, 1987). Algunas consecuencias de esta situación fueron tan evidentes que los medios de comunicación informaron regularmente sobre ellas, aunque sin demasiado interés por confirmarlos de manera sistemática. Aumentaron la desnutrición, especialmente entre los ninos; la mortalidad infantil, debido a la desnutrición y enfermedades infecciosas; los porcentajes de deserción escolar, de divorcio y delincuencia incluyendo el abuso de menores. Consecuencias menos espectaculares y también menos difundidas, pero que no son extrañas a los demógrafos son el aumento de la participación de mujeres y niños en la mano de obra, menos matrimonios, hogares más grandes, tasas de nacimiento más bajas y cambios en los patrones migratorios.

\section{La crisis esquiva}

Es bastante más difícil formular una respuesta precisa que se aplique en forma general a la pregunta de cómo afectan las crisis económicas los fenómenos demográficos que los que los informes de medios de comunicación antes mencionados sugieren. Como veremos posteriormente, los periodos de depresión en economías semindustrializadas e industrializadas, aun en el Tercer Mundo, tienen efectos sustancialmente diferentes de los que caracterizaron las crisis de subsistencia de la Europa preindustrial.

Aun en una región relativamente homogénea como la América Latina contemporánea, la recesión mundial de comienzos del decenio de 1980 ha tenido consecuencias diversas. Para comenzar, las crisis institucionales y económicas afectaron a los países de América Latina. Chile, por ejemplo, apenas había atravesado por una serie de reformas económicas estructurales que dejaron su comercio extremadamente vulnerable a las fluctuaciones. Tam- 
bién Costa Rica fue castigada severamente. Por otra parte, Colombia tuvo sólo una leve recesión y Panamá no sufrió consecuencias. En segundo lugar, las medidas institucionales que varían de pais a país modificaron los efectos demográficos de estas tendencias económicas. Asi, por ejemplo, Chile tiene un complejo sistema de asistencia nutricional que atenuó el efecto recesivo sobre la salud infantil, pero la mayoría de los países carecen de un sistema similar. Finalmente, al menos en teoría, debería distinguirse entre los efectos de la recesión que precedieron a los ajustes estructurales que se hicieron en la mayor parte de la región y los efectos de estos ajustes. La conjunción de ambos procesos es lo que le da carácter de crisis económica a la depresión de los años ochenta. Sus efectos pueden ser diferentes, como se ha demostrado en el caso de la dinámica de la mano de obra (véase Lavell, 1988). Sin embargo, es casi imposible hacer una distinción sistemática en variables demográficas porque son escasas las fuentes de datos y hay muchos vacíos; no intento aquí hacer tal distinción.

A pesar de la aparente irrefutabilidad de las cifras que proporciona Folha de São Paulo, la evaluación de las condiciones de salubridad, los niveles de nutrición, y especialmente de los factores demográficos como mortalidad infantil, fecundidad, patrones matrimoniales y migración como indicadores del impacto de la depresión económica, está llena de trampas metodológicas que no siempre aprecian los especialistas en las consecuencias sociales de la crisis de la deuda en América Latina.

Un estudio de la UNICEF relacionó los índices de mortalidad infantil con la recesión de Brasil a comienzos de los años ochenta. En el noreste, la mortalidad infantil aumentó de 93.1 por mil niños de menos de un año de edad en 1982 a 116.1 en 1984 , es decir un incremento promedio del $25 \%$. El aumento de la mortalidad infantil en ese periodo fue de $12 \%$ para todo Brasil. La Organización Panamericana sobre la Salud (PAHO) informa que los casos de malaria en Brasil aumentaron de 197000 en 1981 a 378000 en 1984. Algunos informes confirman que en Brasil reaparecieron la peste bubónica y la lepra y no solamente en las áreas rurales. Brasil es uno de los paises que tiene la mayor cantidad de casos de SIDA en el mundo y se está propagando rápidamente. Muchos observadores brasileños ven un vinculo claro entre el deterioro social y la deuda. El servicio de la deuda ha vaciado recursos de los gastos del sector público para el servicio social. La cafda constante en los estándares de educación y de salud ha condenado a gran parte de la población a niveles graves de malnutrición y más vulnerabilidad a las enfermedades contagiosas (Roett, 1987: 21-23). Debemos tomar con reserva tales informes; no es necesario decir que son 
escasos los datos estadísticos confiables sobre los cuales se deben basar las conclusiones.

Pocos países en América Latina tienen un sistema de registro civil completo y bastante eficiente para publicar cifras sobre mortalidad y fecundidad actualizadas y precisas. Los métodos de estimación indirectos y convencionales de ahora se desarrollaron para analizar niveles y tendencias a largo plazo, pero no ayudan a detectar las fluctuaciones a corto plazo. Aun las preguntas en apariencia directas como, por ejemplo, si la mortalidad infantil aumentó recientemente o no -es el caso de las cifras de la UNICEF- están por lo general en discusión. Es difícil obtener cifras de migración interna, aun en países desarrollados. Con raras excepciónes, se necesitan datos de censos o.encuestas que pueden no existir o estar retrasados. En América Latina, los datos confiables de migración deben obtenerse de los censos, que en la mayoría de los países no van más allá de 1982. Además, como sugieren Martine, Neiva y Macedo (1984), una de las consecuencias de disminución del gasto público en países como Brasil ha sido precisamente la reducción en la serie de datos migratorios los cuales, al contrario de las estadísticas sobre empleo y salarios, están disponibles para el decenio de 1970, pero virtualmente no existen para el de 1980. Por lo tanto, las conclusiones sobre estos temas tienen que basarse en datos completamente impresionistas o parciales.

Aun cuando se disponga de datos recientes, un problema común consiste en la imposibilidad o el fracaso para obtener datos apropiados para su comparación. Los datos que se refieren a sólo un punto en el tiempo, por muy impresionantes que sean, en el mejor de los casos dan una visión parcial. Como Sawyer (1989) subraya, es importante darse cuenta de que los periodos de crisis en los paises del Tercer Mundo no son momentos atípicos en los esquemas normales de prosperidad y crecimiento sostenido. Desempleo, ingresos bajos, desnutrición e índices de mortalidad infantil elevados son parte de la realidad de todos los días aun en periodos de crecimiento económico. El índice de mortalidad infantil de $10 \%$ mencionado en Folha de São Paulo es sin duda alto, pero no significa que haya aumentado recientemente, si tenemos en cuenta que la mortalidad infantil en Belo Horizonte (no sólo en las favelas) a principios de los años setenta, culminación del "milagro económico" brasileño, tuvo índices del orden de 125 por 1000 (Wood, 1977). Así también, la desnutrición es un problema serio en muchas partes de América Latina, pero las series de datos son muy escasos para demostrar que el fenómeno se debe realmente a la crisis de la deuda. 
Otro reto es separar los efectos demográficos de la crisis provocada por la deuda de procesos estructurales a largo plazo. Cuando estas tendencias son opuestas, como debería esperarse, por ejemplo con respecto a la mortalidad, esto no representa un obstáculo serio. Sin embargo, en el caso del aumento de índices de delincuencia, divorcio, participación de mano de obra femenina, disminución de la fecundidad y migración hacia las ciudades más importantes, el fenómeno a corto plazo se puede entrelazar con tendencias seculares hasta el punto de que puede ser en extremo difícil identificarlo.

El regreso gradual de la lepra a Brasil, que comenzó por lo menos a principios de los años setenta, pudo o no haberse fortalecido por reducciones en el presupuesto de salud desde 1980. Así también, la propagación de la malaria (560 000 casos en 1988) y del SIDA es un hecho, pero las causas de estas dos tendencias son bastante especificas y están relacionadas sólo indirectamente con la deteriorada situación económica del país o sus instituciones de salubridad.

Los desfases son otro factor que debe tenerse en cuenta. Las recesiones económicas pueden tener una influencia inmediata en los índices de matrimonios, pero necesitan por lo menos un año (o casi) para registrar algún efecto en los índices de natalidad. De acuerdo con el estudio clásico de Brenner [1973), en periodos de inestabilidad económica, en Estados Unidos, la mortalidad fetal tiende a incrementarse en los meses posteriores a los primeros signos de recesión económica y la mortalidad perinatal después de casi un año, pero los índices de mortalidad posnatal pueden registrarse entre los tres y cinco años. Irónicamente, en el momento que apareció el artículo en el Folha de São Paulo, la mortalidad infantil en el estado reflejaba la tasa más baja (41.9\% oo entre mayo de 1983 y abril de 1984). En el segundo semestre de 1984, cuando el empleo mostraba los primeros signos de recuperación, la mortalidad infantil aumentó en el estado.

Finalmente, hay que tomar en cuenta que existen mecanismos neutralizadores que pueden impulsar las tendencias demográficas en direcciones inesperadas. Por ejemplo, al enfrentarse a la perspectiva de deterioro, los segmentos más vulnerables de la mano de obra urbana pueden volver al campo, buscando refugio en la agricultura de subsistencia; debido a eso, disminuyen algo las consecuencias de la recesión en la economía urbana. Por otra parte, el deterioro de las condiciones de salubridad en la zona metropolitana de San Pablo durante el auge económico de fines de los años sesenta y comienzo de los setenta fue producto, en parte, de la misma prosperidad del área, ya que la migración intensa desde 
áreas de mortalidad elevada causó una expansión rápida e incontrolada de la periferia urbana (Leser, 1975).

La dificultad para identificar las consecuencias específicas de la situación económica a corto plazo sobre las tendencias demográficas es también una consecuencia de la orientación actual de la teoría demográfica. Por una parte, especialmente con respecto a los países del Tercer Mundo, ha influido en los demógrafos el modelo de transición demográfica, y por otra, la necesidad de implantar políticas específicas para reducir la fecundidad y mortalídad.

Aunque contrarias en muchos puntos de vista, estas orientaciones comparten un énfasis común en el estudio de tendencias a largo plazo, en las que los factores circunstanciales son solamente perturbaciones momentáneas, lo que se refleja en los instrumentos empleados. "Cuando analizamos simultáneamente transformaciones seculares y fenómenos a corto plazo, nuestro bagaje de técnicas analfticas favorece abrumadoramente lo primero" (Hogan, 1985). Corniar (1984) subraya también el mismo punto; en su reseña para UNICEF de literatura reciente, sobre los efectos de la recesión mundial en el bienestar de los niños, tuvo que apoyarse especialmente en estudios de cortes transversales de condicionantes a falta de datos sobre tendencias longitudinales. Los peligros de confundir estos marcos de referencia tan distintos son muy conocidos (Hakkert, 1984). Considerando los índices diferenciales de mortalidad regional dentro de Brasil en 1970, por ejemplo, Wood y Carvalho [1988: 100) mostraron la enorme brecha en la esperanza de vida entre el grupo de ingreso más bajo [ingresos familiares de menos de 150 cruzeiros) del noreste central urbano (40 años) en un extremo, y el grupo de ingresos más elevado (más de 500 cruzados) del sur urbano [67.1 años), en el otro extremo. Comparadas con tales disparidades, las fluctuaciones causadas por oscilaciones económicas han sido notablemente pequeñas. Sin embargo, hay varias razones de por qué no se debería esperar que las fluctuaciones en la mortalidad reproduzcan patrones de corte transversal.

1. Las fluctuaciones de ingresos dentro de las clases sociales producidas por circunstancias económicas a corto plazo son probablemente mucho más pequeñas que los índices diferenciales de ingresos entre regiones y clases, especialmente en Brasil, cuya distribución de ingresos es notoriamente desigual.

2. Se ha mostrado en otros medios [Glewwe y De Tray, 1988) que una proporción significativa de la población no está afectada directamente por programas de ajuste estructural tales como los que se hicieron en el decenio pasado, especialmente en la agricul- 
tura de subsistencia. Este sector es menos importante en América Latina que en Costa de Marfil, donde se llevó a cabo el estudio ya mencionado; es probable que sea cierto en Brasil donde el impacto del costo de transición o ajuste recae sobre la clase media, la cual es un segmento de la población no sólo pequeño comparativamente, sino que goza también de niveles de ingreso en los que incluso las variaciones de ingresos bastante grandes probablemente no influyen en la mortalidad en un grado significativo.

3. Los f́ndices diferenciales de ingresos de cortes transversales son principalmente relevantes como causas determinantes de mortalidad dado que sirven como sustitutos de accesos diferenciales para determinados artículos y servicios, tales como infraestructura sanitaria y educación adquirida. Varios de estos atributos no son fácilmente transferibles, de manera que aquellos quienes tienen acceso a los mismos en épocas de prosperidad continúan disfrutando de sus beneficios en épocas de adversidad económica. Esto es especialmente cierto en lo que se refiere a la educación, pero las medidas sanitarias básicas son lentas para deteriorarse como consecuencia de las oscilaciones económicas.

Debería concluirse por lo tanto, que los estudios de cortes transversales de variaciones en la mortalidad y en otros procesos demográficos entre estratos sociales que se han vuelto bastante comunes en años recientes, tienen una relevancia muy limitada para el entendimiento del impacto demográfico que producen las oscilaciones económicas.

De otra manera, Sell y Kunitz (1987) han intentado juntar evidencia de corte transversal para la hipótesis acerca del problema de la deuda que afectó el lento ritmo de la declinación de la mortalidad en el Tercer Mundo durante los años setenta. Como concepto correlativo, la esperanza de vida aumenta en 62 paises subdesarrollados entre 1970-1975 y 1980-1985 con varias dimensiones de la carga de la deuda de estos países en 1970 y 1982 . Aunque las relaciones son débiles, llegaron a resulłados estadísticamente significativos y consistentes. De acuerdo con sus análisis, los aumentos en esperanza de vida fueron negativos en forma relativa a los niveles de deudas per cápiła, pero de manera positiva con aumentos de la deuda externa per cápita. La interpretación es que los países muy endeudados, que tuvieron que reducir el gasto social, sufrieron consecuencias negativas en términos de su habilidad para reducir la mortalidad. Por otra parte, los países que aumentaron sus deudas a través de nuevos préstamos pudieron canalizar algunos de éstos en proyectos que favorecieron el aumento en la esperanza de vida. 
A pesar de estos resultados bien definidos, este autor no está completamente convencido de que todas las variables confusas posibles se eliminaron del análisís. Debería notarse, por ejemplo, que existe una correlación moderada entre la deuda per cápita y el PIB per cápita y la esperanza de vida de 1970-1975 por ejemplo, para comenzar, los países con la deuda per cápita más elevada eran los más ricos y con las esperanzas de vida más elevadas. En un nuevo análisis de 59 de los 62 países, ${ }^{2}$ ambas correlaciones excedían 0.45. Por otra parte, para ambos la esperanza de vida de 1970-1975 y especialmente el PIB per cápita estaban negativamente asociados con los aumentos de la esperanza de vida. Éste es el efecto asintótico, debido al cual los aumentos en la esperanza de vida tienden a disminuir en los países de ingresos elevados, en donde la mortalidad ya es baja. Esto lo mencionan Sell y Kunitz, pero se descarta luego de un análisis preliminar. Sin embargo, solamente estas asociaciones serían suficientes para explicar la relación negativa aparente entre deuda y aumento de esperanza de vida. En una regresión alternativa, la cual utilizó la deuda como un porcentaje del PIB, en lugar de en una base per cápita, e incluyó el PlB per cápita en sí mismo como una variable explanatoria separada, sólo el último adquirió importancia, mientras que el coeficiente estandarizado del primero estuvo cercano a cero.

\section{Crisis demográfica y control social}

La mayoría de los estudios sobre el impacto demográfico de situaciones de crisis agudas se van a encontrar en las áreas de demograf́a histórica o historia económica, en lugar de en los trabajos de demógrafoş contemporáneos. Hasta cierto punto, esto se debe a que el beneficio de la retrospectiva hace más fácil separar la interrelación de tendencias a corto plazo y seculares. Sin embargo, la principal razón es la gran proporción de crisis de población en el pasado. Las grandes hambrunas a comienzos del siglo XIV y las subsiguientes devastaciones de la peste bubónica son los mejores ejemplos conocidos de un desastre de población que diezmó las poblaciones de diversas partes de Europa, un daño que llevó casỉ dos siglos para repararlo. Varias crisis de población menores ocurrieron durante el siglo XVII en distintas partes de Europa (Meuv-

\footnotetext{
${ }^{2}$ Los datos de esperanza de vida se obtuvieron de las mismas fuentes que en el análisis original, pero debido a un acceso menos completo a datos de deuda, tuvieron que eliminarse tres países y algunas diferencias se notaron con respecto a los datos de los países restantes.
} 
ret, 1965; Skipp, 1978). La hambruna de la papa en Irlanda del siglo XIX es un ejemplo más reciente de una crisis de subsistencia que tuvo repercusiones demográficas importantes.

Sin embargo, hay diferencias significativas entre estas crisis históricas y los efectos de las recesiones económicas contemporáneas, ambas en términos de sus causas y de sus consecuencias demográficas. En las economías predominantemente rurales de Europa hasta el siglo XVIII, los factores demográficos y ecológicos, en especial las malas cosechas y las presiones de la población maltusiana, fueron las causas principales de las epidemias y las crisis de subsistencia, especialmente aquellas de los siglos XIV y XVII. En contraste, las recesiones del siglo XX se basaban generalmente en la economía urbana (la economía de subsistencia actuó por lo general como una especie de válvula de seguridad). En términos de las consecuencias, las crisis demográficas de la Europa preindustrial reflejan un grado mucho menor de control social, aun en comparación con el Tercer Mundo actual, sobre los componentes demográficos principales que podrían ser afectados: migración, matrimonio, fecundidad y mortalidad. Los dos primeros, emigración y casamiento postergados, constituyen evidentemente los mecanismos tradicionales por los cuales las poblaciones del pasado respondían a las situaciones de crisis, aunque no siempre con los resultados deseados. De acuerdo con los historiadores y los demógrafos históricos (Meuvret, 1965), la emigración masiva de áreas de depresión económica fue exactamente uno de los mecanismos que exacerbó la crisis demográfica del pasado, dado que era el factor principal que permitió que la epidemia se esparciera como resultado de las crisis de subsistencia.

El síntoma más claro de tales situaciones fue un aumento elevado en la mortalidad, hasta el punto de que los demógrafos históricos definen actualmente una crisis demográfíca como "un exceso de muertes sobre nacimientos en un área dada durante un periodo especifico" (Sogner, 1979). Dependiendo de si otros procesos demográficos también fueron afectados, las crisis se clasifican a veces ya sea como puramente epidémicas, como en el caso del aumento de índices de muertes sin cambios en otros procesos demográficos, o de subsistencia, por ejemplo, en el caso de disminuciones concomitantes de la fecundidad, matrimonios postergados o emigración.

No existe un acuerdo acerca del grado de disminución de la fecundidad, misma que acompaña generalmente las crisis de subsistencia constituyendo una respuesta consciente hasta cambiar las condiciones económicas. De acuerdo con algunos autores (Chaunu, 1971), la población intentó reducir su descendencia bajo 
estas circunstancias, a través del coitus interruptus o aborto inducido. Para otros (Dupâquier, 1972), la declinación de las tasas de nacimiento durante las crisis de subsistencia tuvieron causas fisiológicas o de otra manera, no intencionales: aumentos de la mortalidad materna, actividad sexual disminuida, esterilidad por desnutrición y abortos espontáneos.

En contraste, las respuestas demográficas a las crisis del siglo $\mathrm{xx}$ se caracterizan por un espectro más amplio de mecanismos de control social. La fecundidad se ha vuelto sujeto de control individual difundido, y es ahora uno de los mecanismos de regulación principales рог los cuales las poblaciones se adaptan a sí mismas para cambiar los prospectos económicos. La mortalidad está sujeta a los controles colectivos de medidas sanitarias básicas, medidas de salud públicas, e intervención nutricional, al igual que a educación individual y conciencia de la higiene, hasta el grado de que ahora lo hace relativamente insensible a cualquiera con excepción de los desastres económicos más severos. La mortalidad infantil se mantiene firme por lo general como un primer indicador de la calidad general de la vida en una población. Sin embargo, existe ahora un acuerdo que no responde directamente a deterioros a corto plazo en las condiciones de vida y puede ser controlado hasta un límite considerable. Muchos de los controles no están afectados en forma inmediata por reducciones en el gasto social o por condiciones de vida deterioradas. La higiene pública básica existente puede evitar que la mortalidad infantil aumente mucho después de que las inversiones en esta área se hayan suspendido. Los programas de asistencia nutricional con objetivos claros dirigidos a los grupos de población más vulnerables pueden evitar problemas agudos de desnutrición.

Las muertes de infantes a causa de diarrea se pueden mantener a un mínimo ahora que se ha comprobado que la terapia de rehidratación oral es una cura efectiva y de bajo costo (UNICEF, 1987).

Esto no implica que las sociedades contemporáneas estén inmunes a las consecuencias de salubridad de las recesiones. MacMahon y Yen (1971) han demostrado que ciertas malformaciones congénitas en Estados Unidos se incrementaron durante los años treinta y el estudio antes mencionado por Brenner (1973) revela un retardo de los diferentes componentes de la mortalidad infantil durante los periodos de desempleo más elevados a partir de los años treinta. En lugares del Tercer Mundo, en donde las condiciones de salubridad son especialmente precarias, las crisis de mortalidad todavia suceden hasta hoy. Sogner (1979) cuando cita a Fernando, menciona la epidemia de malaria de 1934-1935 en Sri Lanka que azotó a una población debilitada y desnutrida después 
de la depresión económica de 1930-1934 y elevó los indices de mortalidad infantil hasta más de $400 \%$ oo en algunos distritos. Sen documentó (1981) otros ejemplos de hambrunas y su consecuente mortalidad en Bengala (1943) y Bangladesh (1974), y más recientemente los demógrafos han documentado sistemáticamente estudios de las consecuencias demográficas de las hambrunas (Bongaarts y Cain, 1982; Hugo, 1984; Kane, 1987).

Sin embargo, las hambrunas son sólo el principio de una serie de posibles secuelas de la paralización de los mecanismos de soporte social. Como regla, las crisis económicas del siglo $\mathrm{XX}$ en los países industrializados y semindustrializados no han tenjdo tales consecuencias extremas, y en general su efecto sobre los porcentajes de muertes ha sido sorpresivamente modesto. La gran depresión de los años treinta en Estados Unidos causó $46 \%$ de caída en el PIB entre 1929 y 1933, en lo más profundo de la depresión, mientras que el desempleo aumentó de 0.4 a $11.5 \%$ de la población total de más de 10 años. La migración internacional llegó a una paralización abrupta, de 279678 en 1929 hasta 23068 en 1933. Las tasas de matrimonios también respondieron rápidamente, cayendo desde $10.1 \%$ oo en 1929 hasta el 7.9 en 1933, después de lo cual alcanzaron rápidamente niveles de pre-depresión. La tasa bruta de natalidad descendió hasta el 16.69 oo en 1933, una baja histórica que no se volvió a presentar hasta los años setenta. Sin embargo, la tasa bruta de mortalidad aparentemente no se afectó; cayó desde $11.9 \%$ oo en 1929 hasta 10.7 en 1933, después de lo cual continuó fluctuando de manera no sistemática entre 10.6 y 11.6, hasta fines de la década. La tasa de mortalidad infantil, considerado generalmente un indicador más sensitivo de las condiciones de vida generales, bajó de $67.6 \%$ oo en 1929 a 57.6 en 1932, se elevó apenas hasta 60.1 en 1934, y luego continuó su tendencia descendente. Si no fuera por los porcentajes particularmente bajos, registrados en 1932 y 1933, la tendencia hubiera sido una declinación continua.

Desde el momento en que las estadísticas vitales disponibles permiten cualquier comparación con América Latina durante este periodo, las conclusiones son bastante similares. Con excepción de México, la mayoría de los países de América Latina sufrieron una marcada aunque temporal baja en las tasas de nacimiento debido a la crisis económica de los años treinta [Collver, 1965). En algunos países, como Argentina y Chile, las tasas de nacimientos se mantuvieron relativamente bajas durante varios años, pero eventualmente se elevaron nuevamente. Las tasas de mupcialidad entre 1929 y 1933 bajaron hasta 19\% en Argentina, 25\% en Uruguay y $33 \%$ en Chile, pero se recuperaron rápidamente con pos- 
CUADRO 1

Chile: porcentaje de mortalidad infantil desde 1970-1985.

\begin{tabular}{llll}
\hline & $0 / 00$ & & $0 / 00$ \\
\hline 1970 & 82.2 & 1980 & 33 \\
1971 & 73.9 & 1981 & 27 \\
1972 & 72.7 & 1982 & 23.6 \\
1973 & 65.8 & 1983 & 21.9 \\
1974 & 65.2 & 1984 & 19.6 \\
1975 & 57.6 & 1985 & 19.5 \\
1976 & 56.6 & 1986 & 18.6 \\
1977 & 50.1 & & \\
1978 & 40.1 & & \\
1979 & 37.9 & & \\
\hline
\end{tabular}

Fuente: Chile, Instituto Nacional de Estadística (INE), Anuario Demográfico 1985.

terioridad. También ocurrió en Colombia una abrupta baja de las tasas de nupcialidad. Sin embargo, hay una pequeña evidencia de un aumento sistemático de la mortalidad infantil a lo largo del continente, aunque en Colombia se dieron incrementos aparentemente menores durante la primera mitad de los años treinta y en Argentina durante la segunda mitad.

Esta ausencia de grandes aumentos en la mortalidad durante periodos de depresión económica no implica que las crisis modernas no tienen impacto de ninguna clase sobre las condiciones de salud o mortalidad. MacMahon y Yen (1971) han demostrado que durante los años treinta se registraron aumentos en ciertas malformaciones congénitas en Estados Uniclos. El estudio antes mencionado por Brenner (1973) revela un descenso en los diferentes componentes de la mortalidad infantil durante periodos de alto desempleo a partir de los años treinta. Por otra parte, Eyer y Sterling (1977) sostienen que las tasas de mortalidad adulta en Estados Unidos durante el siglo pasado disminuyeron más rápidamente durante las recesiones económicas. Aunque el suicidio y algunas otras causas violentas de muerte tienden a elevarse durante los periodos de alto desempleo, las enfermedades degenerativas que responden por la mayor parte de la mortalidad en los adultos son más sensibles al stress, a las horas prolongadas de trabajo y a la desintegración social característica de los periodos de desarrollo rápido.

\section{Vida y muerte bajo la austeridad}

Volviendo a la crisis de la deuda de los años ochenta, una comparación de las tendencias de mortalidad y salubridad en Chile, Cos- 
ta Rica y Brasil, muestra un panorama de alguna manera contradictorio y confuso. El caso chileno en particular ha sido objeto de un cuidadoso estudio. Por una parte, esto se debe a que las estadísticas demográficas chilenas son de mejor calidad que las de la mayoría de los países de América Latina. Por otra parte, se ha despertado el interés por la curiosa circunstancia de que casi una década de economías de mercado libre, con la exacerbación concomitante de desigualdad social, niveles de consumo declinantes de los segmentos de población más pobres [Cortázar, 1983; Riveros y Labbé, 1984), y hasta una caida de $14.4 \%$ del GDP en 1975 , han fallado en la interrupción de la tendencia de mortalidad infantil descendente del país. Esto es todavia cierto a comienzos de los años ochenta, época en que el GDP bajó nuevamente a un espectacular $17.2 \%$ entre el último semestre de 1981 y el segundo semestre de 1983, mientras que el desempleo alcanzó los porcentajes más elevados que en cualquier otro lugar del continente (Foxley y Raczynski, 1984).

Sin embargo, como se indica en el cuadro 1, la tasa de mortalidad infantil mantuvo su ritmo rápido de declinación, desde $82.2 \%$ oo en 1970 hasta $19.5 \%$ oo en 1985 . Es aparente algun retardo debido a lo profundo de la reciente recesión en 1982, pero teniendo en cuenta que la tasa ya se está acercando al límite teórico de reducciones posibles, dicho retardo debería esperarse aun en circunstancias normales. De acuerdo con Haignere (1983), la causa de esta tendencia inesperada deberfa buscarse en el aumento de gastos para la salud infantil y materna y en los programas de nutrición realizados por la junta militar la cual evitó un deterioro de otra manera inevitable en las condiciones de salud de los niños e infantes. Los investigadores del CIEPLAN de Chile [Raczynski y Oyarzo, 1981; Foxley, Ski, 1984; Raczynski, 1985) llegaron, en esencia, a la misma conclusión. Éstos también señalaron el desigual énfasis que se puso en estos programas cuando los gastos de salubridad totales per cápita habían disminuido hacia 1970. Castañeda (1984) y Solimano y Haignere (1983), también reconocen alguna influencia del mejoramiento en la educación y una distribución favorable de los niños por el orden de nacimiento.

La situación chilena es aún más incomprensible cuando se le compara con la de Costa Rica, país conocido por su vieja tradición en el cuidado de la salud. Un estudio estadístico de la mortalidad infantil en Costa Rica desde 1910 hasta 1981 realizado por Rosero (1983) detecta un aumento sistemático en las tasas de mortalidad infantil en un año posterior al comienzo de las recesiones históricas. El estudio no abarca los efectos de 1982, pero existen cifras recientes que muestran que sí tuvo lugar un pequeño 
aumento del $18.1 \%$ en 1981 a 18.8 en 1982. En 1983 y 1984, la tendencia se recuperó, aunque a una tasa menor que antes. A pesar de la reducción en los programas de nutrición infantil, las estadísticas del Ministerio de Salud de Costa Rica muestran que la desnutrición entre los niños prescolares disminuyó desde $8.6 \%$ en 1978 hasta $4.1 \%$ en 1982 . No hay estadísticas, año con año, de los años subsiguientes, pero hay reducciones significativas de datos relativos al consumo de la comida y al cuidado de la salud para la población en general en 1982. El hecho de que la tasa de mortalidad infantil haya reaccionado más claramente puede deberse al aumento de la lactancia materna, desde $7.4 \%$ en 1978 hasta $90.4 \%$ en 1982 (Mata et al., 1984) y a la recuperación relativamente más rápida de varios de los elementos de salud mencionados previamente en 1983 y 1984.

A pesar de la ausencia de un incremento similar en la mortalidad infantil de Chile, existen indicaciones de que no todo está bien. La menor disminución tuvo lugar entre 1973-1974 y entre 1975-1976. La primera pudo suceder por la desorganización social que siguió al golpe militar de 1973 y la última a la recesión de 1975-1976. Las cifras se vuelven más claras si se dividen por los niveles educativos de las madres, como lo hicieron Foxley y Raczynski (1984). Así, se nota un aumento de 144.6\% en 1973 y 157.3 en 1974 , y nuevamente de $133.5 \%$ en 1975 a 136.5 en 1976, en el caso de madres sin educación formal. Algo inesperado fue también en estos años un leve aumento de madres con educación media o superior. Otra indicación es el aumento de la mortalidad (en niños de 1 a 4 años) de $2.49 \%$ en 1973 a 2.78 en 1974 , y de $1.09 \%$ en 1982 a 1.22 en 1983 (Raczynski).

Durante muchos años, la tasa de mortalidad infantil se ha tomado como un indicador de la calidad general de vida de una población. Sin embargo, existe ahora un acuerdo entre los expertos en cuanto a que las tasas de mortalidad infantil no responden directamente a deterioros a corto plazo en las condiciones de vida y pueden, hasta cierto punto, estar controlados por políticas en higiene pública, cuidado de la salud y nutrición. El caso chileno es una ilustración vívida de este punto. Sin embargo, la sensibilidad del indicador de mortalidad infantil responde sobre el capital de salud acumulado; ya sea individual o socialmente no existe razón por la que un deterioro del estándar de vida de la clase media debería tener un efecto inmediato en las tasas de mortalidad infantil; esto no es necesariamente cierto para aquellos segmentos de la población que sobreviven en el nivel de subsistencia. En la misma forma, el alcance difundido de la higiene pública puede recorrer un largo camino para evitar un repunte en la mortalidad infantil 
durante una situación de recesión, pero es poco probable que una campaña de emergencia cambie el curso por sí misma para evitar el esparcimiento de determinadas enfermedades infecciosas por medio de una inmunización masiva.

Los resultados no se alcanzan con éxito dentro de un periodo corto. Son los resultados de un proceso de por lo menos tres décadas de esfuerzo continuo, y sólo de esta manera uno logra que la suma de medidas, programas e intervenciones disminuya los efectos negativos de las crisis económicas periódicas (Monckeberg et al., 1984: 13, citado en Raczynskin, 1985).

En este contexto, es importante destacar la tradición de largos años de los programas de nutrición y de salud chilenos. En Brasil, estas políticas son mucho más recientes y no de tan largo alcance. Una circunstancia curiosa es que las tendencias de la mortalidad infantil en Brasil a partir de los años sesenta, analizadas en periodos de varios años, muestran un esquema contra-cíclico con respecto al crecimiento económico. Aunque la evidencia no es completamente concluyente, existen fuertes indicaciones acerca de que la mortalidad infantil en los ceniros urbanos más importantes aumentó durante el periodo de rápido desarrollo de 1968-1973. Entre otros factores, este incremento se ha atribuido al deterioro del salario legal mínimo y a la insuficiencia de los proyectos de higiene pública para ir al mismo paso del crecimiento migratorio en la periferia urbana (Wood, 1977). Las autoridades percibieron la necesidad de que hubiera más inversiones en los programas sociales y de higiene pública. Particularmente, los últimos se expandieron considerablemente durante los años setenta [Vetter, 1983) y se transformaron en uno de los principales factores subyacentes a la declinación de la mortalidad infantil del periodo de 19751979, cuando paradójicamente la economía ingresó a una fase de crecimiento más lento y hasta de recesión. Las campañas de inmunización se expandieron considerablemente también durante los años setenta.

Hacer la evaluación de la evolución reciente de la mortalidad en Brasil es complicado debido a la escasez de estadísticas confiables. Las cifras indirectas de la mortalidad infantil basada en los censos de 1970 y 1980 y en la National Household Survey (PNAD), de 1984, no dejaron duda de que durante estos periodos hubo considerables mejoras de salud en prácticamente todo el país. La mortalidad infantil, según se informa, bajó desde $113.8 \%$ a fines de los años sesenta hasta 87.9 a fines de los años setenta y 68.1 a comienzos de los años ochenta (FIBGE, 1986). De acuerdo con 
CUADRO 2

Proporciones de los nacimientos y muertes de infantes (por 1000 ) que sucedieron y se registraron en al mismo año calendario para Brasil y algunas de sus áreas metropolitanas, 1978-1987

\begin{tabular}{|c|c|c|c|c|c|c|c|}
\hline & Brasil & Fortaleza & Recife & $\begin{array}{c}\text { Belo } \\
\text { Horizonte }\end{array}$ & $\begin{array}{l}\text { Río de } \\
\text { Janeiro }\end{array}$ & $\begin{array}{c}\text { San } \\
\text { Pablo }\end{array}$ & Curitibo \\
\hline 1978 & 81.1 & 136.3 & 131.1 & 80.3 & 66.5 & 73.9 & 62.5 \\
\hline 1979 & 74 & 129.1 & 118.3 & 75.1 & 64 & 65.8 & 56.9 \\
\hline 1980 & 68.1 & 117 & 118.9 & 63.3 & 59.4 & 58 & 50.6 \\
\hline 1981 & 62.2 & 109.2 & 96.3 & 57.7 & 55.3 & 57.4 & 46.7 \\
\hline 1982 & 56 & 92.5 & 92.5 & 60.2 & 50.2 & 55.4 & 41.9 \\
\hline 1983 & 57 & 104.3 & 96.1 & 52.5 & 49.2 & 48 & 40.6 \\
\hline 1984 & 60.9 & 111.3 & 105.3 & 52.3 & 54.3 & 54.3 & 42.1 \\
\hline 1985 & 49.2 & 81.2 & 87.1 & 51 & 45.2 & 41.2 & 38.3 \\
\hline 1986 & 47.4 & 62.8 & 84.7 & 43.8 & 45.3 & 41.4 & 35.2 \\
\hline 1987 & 45.3 & 53.8 & 66.5 & 41.2 & 41 & 38 & 35 \\
\hline
\end{tabular}

Fuente: Brasil, Fundaçảo lBGE. Estatisticas do Registro Civil.

cálculos anuales basados en el PNAD de 1984, realizado por Goldani et al. (1989), el IMR disminuyó, con fluctuaciones menores, desde 76.4 en 1976 hasta el 54.9 en 1983. En las áreas urbanas, en donde uno esperaría un impacto más severo de la crisis, la declinación fue en realidad más aguda, desde 72.0 hasta 44.2.

Otro signo de mejora se ve en el porcentaje de niños desnutridos hasta la edad de 5.5 años en el noreste de Brasil, como lo señala el Demographic and Health Survey de 1986 (BEMFAM/Westinghouse, 1986). Aunque la proporción total de $38.8 \%$ de los niños con algún grado de desnutrición de acuerdo con la clasificación de Gómez es ciertamente alta, se compara favorablemente con un estudio de 1974-1975 realizado por el Instituto Brasileño de Geografía y Estadística, el cual encontró una proporción verdaderamente alarmante de $86.4 \%$ [FIBGE, 1982). En especial se encontró que la desnutrición avanzada había disminuido de $35.5 \%$ a $7.0 \%$. Sin embargo, el hecho de que las dos fuentes de datos no sean comparables directamente ha conducido a muchos observadores a tener el panorama de que en la actualidad la desnutrición infantil puede ser un problema tan serio como a mediados de los años setenta (Silva, 1986).

Desde 1983, la única fuente oficial disponible para datos de mortalidad infantil en Brasil es el sistema de registro civil, mismo que tiene muchas limitaciones en términos de calidad de datos. El cuadro 2 nos brinda una lista de las proporciones de nacimientos y muertes de infantes ocurridas y registradas en el mismo año para el pais en su totalidad y para algunas de sus ciudades princi- 
pales. Estas cifras no son tasas reales de mortalidad infantil, y se debe tener cuidado en su interpretación. En particular, debido a un escaso registro especialmente de nacimientos, éstos no son comparables entre áreas del país. Además, las tendencias a largo plazo pueden estar afectadas hasta cierto punto por un mejoramiento de la cobertura.

A pesar de estas restricciones, la tendencia total pareciera ser la de un dramático descenso en la mortalidad infantil, en especial en el noreste (Fortaleza y Recife), con excepción de 1983-1984, fecha en que ya sucedieron aumentos en la mayoría del país. En un controvertido estudio en circulación realizado por la UNICEF, Becker y Lechtig (1986) interpretaron lo último como el comienzo de una nueva tendencia. En un escrito más reciente realizado por Becker (1988), éste reconoce por qué esta tendencia no duró, él estima las tasas correctas de mortalidad infantil de 1984 y 1985 eran de 73.7 y 66.09 oo respectivamente, para Brasil y de 116.1 y 95.0 para el noreste. En el estado de San Pablo hay una tendencia aparentemente similar en los datos y los registros son bastante confíables. Después de alcanzar su descenso histórico de $41.9 \%$ on en el periodo que va desde mayo de 1983 hasta abril de 1984, la tasa de mortalidad infantil en este estado aumentó temporalmente hasta 45.4 en noviembre, antes de recuperar su tendencia descendente hasta alcanzar 33.4 en mayo de 1988. Desde entonces sucedió un leve incremento hasta 34.0 en septiembre de 1988.

Se puede obtener una mayor evidencia de las estadísticas de mortalidad por causa; en publicaciones anuales del Ministerio de Salud de Brasil (Brasil, distintos años), aunque se debe tener cuidado también en la interpretación de estas cifras por el subregistro.

El cuadro 3 muestra las tendencias de la mortalidad proporcional de algunas causas que se podrían asociar con los efectos de la recesión. Se nota claramente que las infecciones intestinales, responsables de una gran proporción de la mortalidad infantil, han estado en retirada. Lo mismo sucedió en Chile (Raczynski y Oyarzo, 1981). Los investigadores brasileños (Monteiro, 1982) atribuyen generalmente esta declinación a las inversiones masivas en la infraestructura urbana, especialmente el acceso al agua potable realizado durante los años setenta. En el contexto chileno, se ha mencionado este argumento con menos frecuencia, pero también allí la disponibilidad de agua potable se extendió considerablemente de acuerdo con los resultados provisorios del censo de 1982. La rehidratación por vía oral, la cual se convirtió en ampliamente disponible durante este periodo, pudo haber reforzado esta tendencia. La tuberculosis, la neumonía y la gripe también disminuyeron; las dos últimas registraron un incremento leve en 1984. 
CUADRO 3

Brasil: mortalidad proporcional por algunas causas 1977-1985

\begin{tabular}{|c|c|c|c|c|c|c|}
\hline \multicolumn{7}{|c|}{ TODAS LAS MUERTES } \\
\hline & $\begin{array}{c}\text { Deficien- } \\
\text { cias en la } \\
\text { nutrición } \\
\%\end{array}$ & $\begin{array}{c}\text { Infecciones } \\
\text { intestinales } \\
0 \%\end{array}$ & $\begin{array}{c}\text { Tubercu- } \\
\text { losis } \\
\% \%\end{array}$ & $\begin{array}{c}\text { Neumonia } \\
\text { y gripe } \\
0 \%\end{array}$ & $\begin{array}{l}\text { Homi- } \\
\text { cidio } \\
0 \%\end{array}$ & $\begin{array}{l}\text { Suici- } \\
\text { dio } \\
\% \%\end{array}$ \\
\hline 1977 & 1.52 & 7.52 & 1.29 & 6.11 & 1.38 & 0.56 \\
\hline 1978 & 1.59 & 7.39 & 1.18 & 5.93 & 1.38 & 0.49 \\
\hline 1979 & 1.66 & 6.07 & 1.00 & 5.06 & 1.57 & 0.49 \\
\hline 1980 & 1.55 & 5.34 & 0.93 & 4.78 & 1.85 & 0.52 \\
\hline 1981 & 1.43 & 4.81 & 0.85 & 4.64 & 2.03 & 0.54 \\
\hline 1982 & 1.34 & 4.41 & 0.76 & 4.60 & 2.10 & 0.53 \\
\hline 1983 & 1.28 & 4.08 & 0.70 & 4.30 & 2.26 & 0.59 \\
\hline 1984 & 1.50 & 3.95 & 0.69 & 4.34 & 2.44 & 0.61 \\
\hline 1985 & 1.29 & 3.01 & 0.65 & 4.18 & 2.51 & 0.54 \\
\hline \multicolumn{7}{|c|}{ MUERTES EN NINOS } \\
\hline & $\begin{array}{c}\text { Deficien- } \\
\text { cias en la } \\
\text { nutrición } \\
\%\end{array}$ & $\begin{array}{c}\text { Infecciones } \\
\text { intestinales } \\
\%\end{array}$ & $\begin{array}{c}\text { Tubercu- } \\
\text { losis } \\
0 \%\end{array}$ & $\begin{array}{c}\text { Neumonia } \\
\text { y gripe } \\
0 \% 0\end{array}$ & $\begin{array}{c}\text { Peso } \\
\text { bajo } \\
\text { al nacer } \\
\%\end{array}$ & $\begin{array}{c}\text { Saram } \\
\text { pión } \\
0 \%\end{array}$ \\
\hline 1977 & 3.30 & 23.77 & 0.14 & 12.78 & 6.68 & 0.70 \\
\hline 1978 & 3.59 & 23.53 & 0.16 & 12.46 & 6.23 & 0.69 \\
\hline 1979 & 4.56 & 19.95 & 0.12 & 10.23 & 6.60 & 0.79 \\
\hline 1980 & 4.34 & 18.14 & 0.12 & 9.71 & 6.48 & 0.76 \\
\hline 1981 & 4.17 & 16.80 & 0.12 & 10.00 & 6.15 & 0.57 \\
\hline 1982 & 4,12 & 16.22 & 0.11 & 10.45 & 6.06 & 0.50 \\
\hline 1983 & 4.08 & 16.08 & 0.10 & 9.20 & 5.42 & 0.47 \\
\hline 1984 & 4.78 & 15.59 & 0.09 & 9.22 & 5.17 & 0.69 \\
\hline 1985 & 4.47 & 13.41 & 0.09 & 9.16 & 5.81 & 0.36 \\
\hline
\end{tabular}

* No comparable con cifras posteriores, debido a clasificaciones diferentes.

Fuente: Brasil, Ministerio de Saúde, Estatísticas de Mortalidade Fundaçåo IBGE, Anuario Estadístico 1986.

El incremento constante de homicidios en América Latina es causa de inquietud. En Brasil se duplicó la cantidad durante los últimos diez años. Su relación con las políticas de austeridad es de alguna manera leve, excepto en países como Perú, en donde la violencia política se incrementó en forma abrupta como consecuencia de la crisis de la deuda. En este país, los homicidios por motivos políticos, virtualmente no existentes hasta 1980, llegaron a 3587 en 1984 , casi $2 \%$ de todas las muertes registradas (Figallo y Valderrama, 1987). Sin embargo, en Costa Rica, el número de homicidios en 1982-1983 fue menor que en 1980-1981, aunque el número de 
convictos por robo durante el mismo periodo se elevó casi a $70 \%$ (cifras del Ministerio de Justicia de Costa Rica). Los suicidios son más elocuentes y reveladores del estado mental nacional. En Costa Rica, aumentó el número de $0.98 \%$ de todas las muertes en 1982 a $1.40 \%$ en 1983. Un pequeño incremento en Brasil se manifiesta en el cuadro 3. La relación con tendencias económicas aparece con más claridad si los casos están divididos por motivos que se sospechan. De un total de 187 en 1982, el número de suicidios en Brasil atribuidos a problemas financieros aumentó hasta 339 en 1983, en lo más profundo de la crisis financiera, cuando Brasil fue forzado a buscar ayuda en el FMI. En 1984 y 1985 disminuyó de alguna manera, hasta 285 y luego hasta 224 , llegando a una cifra menor de 198 en 1986 durante la euforia del Plan Cruzado. Obviamente, es preciso tener cuidado cuando se interpreten estos números, no sólo debido a que los suicidios resultan menos de $1 \%$ de todas las muertes, sino porque más de la mitad no se pueden clasificar de acuerdo con un motivo bien definido.

Con respecto a la mortalidad infantil, el cuadro 3 muestra que en 1984 hubo un claro aumento de la proporción de muertes que se atribuyen a deficiencias de nutrición. En el caso de niños de menos de un año, este cupo aumentó de 4.08 a $4.78 \%$ y para los niños de 1 a 4 años de 5.02 a $6.00 \%$. En el área metropolitana de San Pablo el porcentaje de muertes de infantes atribuibles a problemas endocrinos y de nutrición (la mayoría deficiencias calóricas de proteínas], las cuales habran sido de $4.4 \%$ en el periodo de junio de 1982 hasta mayo de 1983 , aumentó a $6.8 \%$ durante 1984 . Los datos preliminares de 1985 para el noreste (Becker, 1988) muestran un incremento aún más grande desde 4.3 en $1982,4.6 \%$ en 1983 y 5.6 en 1984 hasta $7.7 \%$. Sin embargo, debido a que no existe un sistema de monitoreo año por año del estado de nutrición, las tendencias actuales de desnutrición durante este periodo son poco claros. Los datos de la ciudad de Santo André cerca de San Pablo, en donde los hospitales de niños y clínicas de salud que registran el estado de nutrición de todos los pacientes muestran una declinación de la desnutrición de los niños de 1 a 3 meses de cerca de $25 \%$ en 1980 hasta $18-20 \%$ en 1981, nivel en el cual se estancó hasta la segunda mitad de 1985 cuando hubo otra declinación, primero hasta 15 y luego hasta $10 \%$ en 1986 (FAISA). Esto indica que, aunque la desnutrición continuó siendo un problema a lo largo de la primera mitad de los años ochenta, su incidencia al menos no aumentó durante los años de austeridad. Aunque toda la zona industrial al sur de San Pablo estuvo muy afectada por el desempleo, ésta es también un área relativamente próspera con reservas más grandes, lo que evitó que la situación se deteriorara aún más. 
El porcentaje de muertes por bajo peso al nacer, en el país en su totalidad no aumentó hasta 1985 , pero en el noreste se incrementó levemente en 1982 , de 4.8 a $5.1 \%$ en 1983 , de 6.8 en 1984 y de $13.7 \%$ en 1985 . Sin embargo, debido a que estos porcentajes son relativos al número de niños fallecidos, es obvio que no se puede saber de forma inmediata hasta qué punto transmiten un deterioro real del estado de nutrición de la madre, especialmente considerando la caída de $18.2 \%$ de la sobretasa de mortalidad infantil en 1985. En Recife, Dias et al. (1985) encontró que el porcentaje de niños recién nacidos con peso por debajo de los $2.5 \mathrm{~kg}$ se ha incrementado de manera abrupta a partir de 1982, en casi la misma proporción que el número de horas de trabajo necesarias para adquirir una ración de comida básiça.

Las enfermedades parasitarias e infecciosas aumentaron en todos lados, en parte debido a la epidemia de sarampión que azotó el pais en 1984. Para todo el país, el aumento de la mortalidad proporcional ocasionado рог esta clase de enfermedades fue bastante leve, de 19.17 en 1983 hasta $19.29 \%$ en 1984 para niños menores de un año, y de 20.96 hasta $21.29 \%$ para niños de 1 a 4 años. Sin embargo, en el área metropolitana de San Pablo, el porcentaje de muertes de infantes debido a estas causas alcanzó 21.21 , siendo que el año anterior fue de 17.36. Para explicar la epidemia de sarampión, Macedo (1984) y otros sugirieron que tres años consecutivos de recesión económica habran comenzado a infligir una pérdida de resistencia en parte de la población infantil. Otra interpretación, atribuye el problema a errores administrativos específicos por parte de las autoridades de salubridad. La tendencia nacional de casos informados pareciera sostener la última hipótesis, debido a que transmite de manera aparente muy poca asociación con las tendencias económicas, mientras que todos los años (1982-1983 y 1987) de recesión se caracterizaron por una incidencia menor que el promedio, más allá del brote más grande de sarampión (75\% sobre el promedio de 1978-1987) sucedido en 1986 en el año del Plan Cruzado, a lo que siguieron dos años de recuperación económica parcial y de poder adquisitivo recuperado temporalmente.

\section{Consecuencias a largo plazo}

De acuerdo con las Naciones Unidas (1986: 75), los fenómenos demográficos están sólo débilmente afectados por ciclos económicos. Si esto es cierto, depende de alguna forma del tipo de análisis y de cómo uno define minuciosamente los "fenómenos demográficos". Como se muestra en la sección anterior, los indicadores de 
mortalidad y salud en los tres países considerados no respondieron hasta el punto de causar cualquier impacto apreciable en el crecimiento de la población. Aun en términos de su significación como indicadores de salud, el impacto fue limitado. Simplemente, la recesión no duró el tiempo suficiente para cambiar las tendencias prevalecientes. En Chile, la tendencia de mortalidad descendente continuó, aunque a un paso más lento. En Costa Rica, el incremento temporal de la mortalidad infantil se revirtió rápidamente cuando el gasto en los programas de nutrición y salud se elevó nuevamente en 1983-1984. Sí la crisis de Brasil hubiera continuado tan aguda como en 1981-1983, es probable que los indicadores de mortalidad y salud se hubieran deteriorado más. Sin embargo, en 1984, ya estaba en camino una recuperación temporal, y en consecuencia la situación mejoró en 1985 y 1986. Uno puede preguntarse cual será el efecto a largo plazo de una secuencia prolongada de tales crisis y recuperaciones parciales. Mientras que los efectos inmediatos de la recesión pudieron haber estado atenuados por el efecto acumulado de las inversiones del pasado tanto en higiene pública como en programas de salud, la necesidad de reducir costos en estas áreas como parte de una política de austeridad a largo plazo puede tener como resultado perspectivas futuras menos favorables. Sin embargo, es más probable que los impactos demográficos permanentes de la crisis económica, estén mediatizados por variables relacionadas con el comportamiento que por impactos directos en la supervivencia y salud. En consecuencia, la nupcialidad, la fecundidad y la migración pueden ser objetos de estudios más relevantes que la mortalidad. La investigación en esta área es más difícil, no sólo porque el nacimiento y especialmente las estadísticas de migreción son más deficientes que los datos de registro de muertes, sino también debido a problemas conceptuales. La fecundidad en la mayoría de América Latina venía descendiendo desde antes de los años ochenta; la separación de los efectos seculares de los de corto plazo no es un asunto trivial. Además, les tendencias han sido bastante precisas en los diferentes países. La declinación principal de la fecundidad durante la década pasada sucedió en Brasil (BEMFAM/Westinghouse, 1986) y en Colombia [CCRP/Westinghouse, 1986), pero no en Argentina, Chile o Costa Rica. En los lugares donde sucedieron las declinaciones principales, los expertos se dividen entre aquellos que buscaron sus causas en la modernización y mejores condiciones de vida y los que, por lo contrario, señalaron los estándares de vida deteriorados y el acceso en aumento a la contracepción.

Mientras que existe una gran evidencia histórica de la declinación a corto plazo de las tasas de nacimiento y matrimonio que nor- 
malmente acompañaron periodos de recesión en los países desarrollados hasta la Segunda Guerra Mundial (véanse Basavarajapa, 1971; Glass, 1938; Kirk, 1960; Pressat, 1969), los efectos permanentes de la adversidad económica prolongada son mucho más difíciles de valuar. La nupcialidad constituye uno de los primeros fenómenos demográficos afectados. La fecundidad, por razones obvias, toma bastante más tiempo para reaccionar. En su análisis de la National Household Survey de 1984 (PNAD) de Brasil, Goldani et al. (1989) advirtió un aumento en las probabilidades del primer casamiento hasta 1980, seguido por una aguda declinación. Contra toda expectativa, esta tendencia es más pronunciada en las áreas rurales que en las urbanas. La Tasa Global de Fecundidad [TGF] cayó de 4.02 en 1979 a 3.11 en 1984, tendencia que fue más pronunciada en las áreas rurales. Sin embargo, es difícil saber hasta qué punto la última puede atribuirse a la crisis económica, considerando que la fecundidad en Brasil había estado bajando desde la década anterior a 1979.

En el área metropolitana de San Pablo, se puede advertir una clara declinación del porcentaje de matrimonios, desde $8.129_{00}$ en el periodo de agosto de 1981 hasta julio de 1982, hasta 6.75 dos años más tarde. La tasa de nacimientos, luego de una lenta declinación hasta 29.69 oo para el periodo.de marzo de 1982 a febrero de 1983 , bajó mucho más rápido durante 1984 y los primeros meses de 1985. Cuando de alguna manera la economía mejoró en el segundo semestre de 1984, las tasas de matrimonio crecieron nuevamente, aunque no en los niveles anteriores a 1982. De la misma manera, las tasas de nacimiento se recuperaron en 1985, siguiendo de cerca el incremento en las tasas de matrimonio nueve meses antes. Sin embargo, en 1986 se volvió a la tendencia descendente de ambos, los matrimonios y los nacimientos, a pesar de la recuperación económica. En marzo de 1988, la tasa de nacimiento de los últimos 12 meses estuvo en su baja histórica de $22.2 \%$, mientras que la tasa de matrimonio fue de $6.26 \%$ oo en enero; éste fue el registro menor desde los primeros años del siglo.

Desde una perspectiva a corto plazo, una implicación interesante de la sensibilidad de las tasas de nacimiento con respecto a los ciclos económicos es que, la recesión puede contribuir, de una manera bastante perversa, a bajar la mortalidad infantil. Este punto lo presenta Hojman (1985) en su análisis econométrico de la mortalidad infantil chilena durante los años setenta. El efecto puede suceder por varias razones:

1. El número menor de nacimientos puede implicar menos presión en los escasos recursos relacionados con el cuidado de infantes (Raczynski y Oyarzo, 1981). Por ejemplo, en el área metro- 
politana de Belo Horizonte, Brasil, una declinación de $17 \%$ en el número absoluto de nacimientos desde 1981 hasta 1985, junto con una mejor distribución de la vacunación por edad, mantuvo los niveles de salud, no obstante que se redujo $44 \%$ el número de vacunas de BCG y antisarampión aplicadas durante el mismo periodo.

2. Si la tasa de nacimiento desciende en forma desproporcionada entre los segmentos de población con más desventajas, los niños que realmente nacen tienen más bajo riesgo promedio de morir que los grupos de niños nacidos antes (Hakkert, 1984).

3. La fecundidad más baja implica generalmente una distribución más favorable de nacimientos por espaciamiento y orden de nacimiento. Trussell y Pebley (1984) calculan el impacto potencial de una distribución de nacimientos mejor sobre la mortalidad infantil, controlada por varias variables socioeconómicas, al 10\% y $21 \%$, respectivamente. En un análisis de la mortalidad infantil de Brasil, en el que se admite que está incompleta - por falta de desagregación por estrato social-, Fernández descubrió (1986) que las tasas de muertes, que parecieron haber declinado considerablemente, en realidad se incrementaron durante la segunda mitad de los años setenta si el efecto de la fecundidad se descartó debidamente.

Desde una perspectiva a largo plazo, la implicación de las fluctuaciones temporales de la fecundidad consiste en que los grupos que entran en las edades de reproducción durante las recesiones se pueden casar con posterioridad y tener sus hijos a una mayor edad, pero como se conoce bien por la experiencia histórica de Estados Unidos y de otros países desarrollados, esto no significa necesariamente que su fecundidad completa será menor. Las fluctuaciones de la fecundidad presente en países como Brasil y su correlación evidente con las tendencias económicas permiten dos interpretaciones. De acuerdo con una de ellas, las políticas de austeridad de los años ochenta han afectado la intensidad de la reproducción, debido a la aceleración de un proceso de declinación de fecundidad estructural que de otra manera hubiera ocurrido, aunque a un paso más lento. El otro punto de vista reconoce la existencia de un "efecto de austeridad" superpuesto sobre una tendencia estructural de declinación de la fecundidad, pero interpreta este efecto principalmente en términos de calendario. $\mathrm{Si}$ esto es correcto, significa que las extrapolaciones de la declinación de la fecundidad actual pueden subestimar en exceso las futuras tasas de nacimiento.

Dado el énfasis teórico de modernización y otras explicaciones estructurales de la fecundidad, la tendencia en la literatura de- 
mográfica consiste en interpretar la declinación de la fecundidad actual en el Tercer Mundo en términos de intensidad más que de calendario. En estudios recientes de los descensos de la fecundidad principales en Colombia [CCRP/Westinghouse, 1986), Costa Rica (Naciones Unidas, 1985) y Brasil [BEMFAM/Westinghouse, 1986) se investigó una amplia serie de factores: desde el conocimiento y la disponibilidad de métodos contraceptivos hasta la urbanización, la educación femenina, y los "factores de motivación", pero no es frecuente la pregunta respecto hasta qué punto se pueden revertir estos "factores de motivación" una vez que las condiciones económicas cambien. Incluso los procedimientos analíticos bastante simples para la interpretación de tendencias de la fecundidad, tales como la descomposición de las tasas de fecundidad por órdenes de nacimiento, rara vez se enuncian para aclarar este tema.

La falta de atención a los efectos del calendario tiene un costo potencial. Los demógrafos de Estados Unidos aprendieron una dolorosa lección en los años cuarenta cuando descubrieron que la aparente tendencia secular hacia una más baja fecundidad en la década anterior se habia perjudicado realmente debido a las circunstancias económicas adversas de la Gran Depresión. Por supuesto, que el baby boom en Estados Unidos no puede explicarse únicamente como resultado de un cambio en el calendario de la fecundidad. Easterlin (1962) y otros (Boyer, 1984) han intentado identificar otras causas estructurales que pueden no aplicarse a la América Latina de hoy; por lo tanto no puede enfrentar un baby boom similar en los años venideros, pero si el hecho de posponer los nacimientos, está sucediendo en países como Brasil, hasta un extremo significativo, debido a los prospectos económicos de la era del FMI, puede afectar profundamente las tendencias futuras de la fecundijdad. Por el momento, los demógrafos brasileños tienen muy poca certeza de lo que está sucediendo realmente y hasta qué punto la espectacular declinación de la fecundidad, a comienzos de los años ochenta, puede extrapolarse hacia el futuro.

\section{Migración}

Como ya se señaló, en un contexto de considerable control social e individual sobre los mecanismos causales de la mortalidad y la fecundidad, los efectos demográficos de la crisis económica tienden a manifestarse más claramente por sí mismos en el nivel relativo al comportamiento. Por lo tanto, cabe esperar encontrar respuestas más claras en el área de migración. Sin embargo, como Martine et al. (1984) señalan, la migración podría ser afectada teóricamente en 
dos direcciones radicalmente opuestas. Por una parte, sería posible que los efectos de recesión de las medidas de austeridad causaran un aumento total en la cantidad de movimientos, especialmente de los pobres, en respuesta a la necesidad de encontrar nuevos medios de subsistencia. Por otra parte, la disminución de oportunidades en la economía urbana y en los proyectos de construcción auspiciados por el gobierno o programas de colonización podrían desalentar dichos movimientos. El resultado eventual dependerá en gran parte de los datos específicos de cada área en particular.

Debido a que es imposible generalizar, la evidencia fragmentaria disponible pareciera indicar que de manera más frecuente, las situaciones de crisis económica tienden a disminuir las tendencias de migración prevalecientes. Con respecto a la migración rural a urbana, se ha notado una inversión de las tendencias prevalecientes en casos tales como la ciudad de México. De manera similar, la prensa de Brasil he escrito acerca del regreso de emigrantes de principales áreas metropolitanas al noreste, como resultado del desempleo urbano (Martine et al., 1984). En el caso de la emigración de frontera a la región amazónica de Brasil, Sawyer [1989) señala varias razones por las que la crisis ha implicado menos expulsión y más retención:

1. La crisis ha desalentado la migración hasta el punto de que mucha de la expulsión durante los años setenta se debió a la modernización de la agricultura en otras regiones de Brasil, por lo cual hay menos capital disponible y menos crédito durante los años ochenta (Martine y García, 1987). La baja en el valor de la tierra ha provocado que los pequeños agricultores del sur no puedan vender sus propiedades y adquirir tierras para los proyectos de colonización en el Amazonas. En su lugar, el estancamiento de la economía rural pareciera haber favorecido la multiplicación de pequeños establecimientos, incluyendo a los aparceros y a las personas dedicadas a la agricultura de subsistencia.

2. La reserva de población rural disponible para migración de este tipo se está agotando rápidamente mientras que el grado de urbanización ha aumentado de 56 en 1970 a $68 \%$ en 1980 .

3. Los factores de atracción dentro de la región del Amazonas han sido en gran medida inversiones públicas y privadas en las carreteras, los proyectos de colonización, los ranchos, las represas y los ferrocarriles para los cuales hay disponibles menos fondos en épocas de retracción económica.

Aun así existen excepciones aquí, como en el caso de los garimpeiros (mineros de oro), quienes invadieron la región en gran- 
des cantidades debido al descubrimiento del oro, los precios elevados, la sequía en el noreste y desempleo en el sureste.

La migración internacional dentro y fuera de la mayor parte de América Latina también parece haber sido afectada de manera negativa debido a una combinación de leyes de migración más duras y prospectos económicos desfavorables en los países receptores. Argentina y Venezuela, con anterioridad receptores de trabajadores de los países vecinos, impusieron efectivamente una legislación restrictiva. Aunque los datos que permiten una evaluación de una legislación no están disponibles, los investigadores creen que la legislación más exigente con los bajos ingresos reales descendentes de los trabajadores en Argentina han reducido grandemente las cantidades de trabajadores sin documentación presentes (Villar, 1984). Durante los años setenta, Venezuela recibió muchos trabajadores indocumentados, especialmente de Colombia pero también de Ecuador, Perú y Chile. En 1980, se dictó el Decreto Presidencial 616 como un medio para registrar más de un millón de extranjeros indocumentados que, se creía, estaban en el país. ${ }^{3}$ Concedió amnistía a los ilegales en el país, pero hizo más fácil la deportación de ilegales en el futuro. La ley pareciera haber producido el efecto deseado. La migración neta se volvió negativa en los años ochenta, tanto que desde marzo hasta septiembre de 1983, hubo 22197 extranjeros más que dejaron el país en comparación con los que entraron [Van Roy, 1984). Para todo el periodo de 1980-1985, la migración externa neta se ha estimado en 126625 . Sólo para los colombianos era de 56858 (Torrealba, 1987).

De la misma manera, fue la nueva legislación, con la disminución de su actividad económica, lo que explicó mejor la rápida inversión.

Una tendencia contraria ha estado en camino por algún tiempo en Brasil. Históricamente, este país no ha sufrido los mismos efectos de la "fuga de cerebros" que Argentina. Sin embargo, la crisis de los ochenta parecería estar cambiando el comportamiento de este grupo y aun de trabajadores menos calificados. Una encuesta de 1987 de 1390 adultos en San Pablo indicó que 34\% había pensado emigrar (Folha de São Paulo, julio 31, 1987). Entre éstos, $57 \%$ mencionó la situación económica difícil como razón principal. Aquellos que realmente salían pueden dividirse en residentes extranjeros de largo tiempo decididos a regresar a su tierra $\mathrm{y}$ brasileños nativos en busca de una mejor forma de vida en cual-

\footnotetext{
${ }^{3}$ Solamente alrededor de 275000 personas legalizaron su estado de residencia.
} 
quier lugar. En los últimos años, los países que emitieron el mayor número de visas de residentes a los ciudadanos de Brasil han sido Italia, Portugal, España y Australia, en ese orden (Lessa, 1987). El fenómeno está atrayendo mucho el interés de la prensa. Por ejemplo, la portada de la emisión de marzo 16 de 1988 del Veia de Brasil, el semanario de mayor tirada, contenía la página titulada "Por qué tantos brasileños se van". Artículos similares en periódicos de negocios como el Senhor señalan la reversión curiosa de las corrientes migratorias tradicionales que ahora hace que cantidades significativas de profesionales brasileños prueben su suerte en Portugal. Sin embargo, el significado numérico de estas corrientes no ha sido hasta ahora valorado con exactitud.

\section{Bibliografía}

Basavarajapa, K. G. (1971). "The Influence of Fluctuations in Economic Conditions on Fertility and Marriage Rates, Australia, 1920 a 1938 y 1946 a 1967", en Population Studies 25 (1).

Becker, Roberto A. (1988). "Brasil: principais causas de mortalidade infantil". Ministerio da Saúde, Brasilia, mimeo.

y Aaron Lechtig (1986). Brazil: ovolução da mortalidade infantil no periodo 1977-1984, Brasilia, Ministerio da Saúde.

BEMFAM/Westinghouse (1986). Brazil Demographic and Health Survey 1986, Preliminary Report, Columbia, MD, Westinghouse Institute for Research Development.

Bongaarts, John y Mead Cain (1982). "Demographic Responses to Famine", en Kenneth M., Cahill (ed.), Famine, Nueva York, Orbis Books.

Boyer, Louis (1984). "Dimensions Démographiques de la crise économique mondiale", Études en Développement, 12:47-48, 51-62.

Brasil, Ministerio Da Saúde. Several years, Estatisticas da mortalidade, Brasilia, Ministerio da Saúde.

Brenner, M. (1973). "Foetal, Infant and Maternal Mortality During Poriods of Economic Instability", en International Journal of Health Services, 3 [2].

Castañeda, T. (1984). "Contexto socioeconómico y causas del descenso de la mortalidad en Chile", Estudios Públicos 28, Santiago, Centro de Estudios Públicos.

CCRP/Westinghouse [1986). Tercera Encuesta Nacional de Prevalencia de Uso de Anticonceptivos. Demografía y Salud, informe preliminar, Bogotá, Corporación Centro Regional de Población.

Céspedes, V. (1985). Costa Rica, recuperación sin reactivación, San José, UNED, Academia de Centroamérica.

Collver, D. Andrew. (1965). Birth Rates in Latin America New Estimates 
of Historical Trends and Fluctuations, Institute of International Studies Research, Series, núm. 7, University of California, Berkeley.

Cornia G., Andrea (1984). "A Survey of Cross-sectional and Time-series Literature on Factors Affecting Child Welfare", en World Development, 12 (3), 187-202.

Charbonneau, Hubert y André Larosse (eds.) (1979). The Great Mortalities Methodological Studies of Demographic Crises in the Past, Dolhain, Belgium, Ordina Editions.

Chaunu, Pierre (1971). La civilisation de l'Europe des Lumières, París.

Chen, Lincoln C. y Alauddin Chowdhury (1977). "The Dynamics of Contemporary Famine", en luSSP, Proceedings of the International Union for the Scientific Study of Population. Population Conference, Mexico, Liege, Ordina.

Dias, L., M. R. Camarano y Aaron Lechtig (1985). "A Drought Recession and Prevalence of Low Birth-weigth in Poor Urban Populations of the Northeast of Brazil", en James P. Grant (ed.), Situação mundial da infancia 1986, Brasilia, UNICEF.

Duarte, Isis (1988). "Crisis, familia y participación laboral de la mujer en la República Dominicana", Ponencia presentada en la Conference on the Demography of Inequality in Contemporary Latin America, University of Florida, Gainesville, Flo.

Dupâquier, Jacques (1972). "De l'animal à l'homme; le mécanisme autorégulateur des populations traditionnelles", Éditions de l'Institut de Sociologie, Université Libre de Bruxelles.

Easterlin, Richard A. (1962). The American Baby-boom in Historical Perspective, Nueva York, National Bureau of Economic Research.

Eyer y Sterling (1977). "Stress-related Mortality and Social Organization", Review of Radical Political Economics, 9 [1].

Fernández, Rogelio E. (1986). "O efeito demográfico da mudança dos padrões de fecundidade nas tendēncias da mortalidade infanto-juvenil: uma proposta metodológica". Ponencia presentada en el Vth National Meeting of the Brazilian Association for Population Studies (ABEP), Aguas de Sẩo Pedro, oct. 12-16.

Figallo, Daniel y Mariano Valderrama (1987). "Pobreza y supervivencia en el Perú", Ponencia presentada en el XIVth General Assembly of ClACSO, Recife, nov. 15-20.

Foxley, A. y Dagmar Raczynski (1984). "Vulnerable Groups in Recessionary Situations, the Case of Children and the Young in Chile", en World Development, 12 (3).

Fundação de Assistencia a Infancia de Santo André [FAISA], varios años, Relatorio anual, Santo André, PAISA.

Fundação Instituto Brasileiro de Geografia e Estatistica [FIBGE] [1987]. Anuario Estatistico do Brasil 1986, Río de Janeiro, Fundação IHGE. (1982). Perfil estatistico de crianças e mães no Brasil 1974-1975, Río de Janeiro, Fundação IBGE.

(1986). Perfil estatistico de crianças e mães no Brasil, aspectos socio-económicos da mortalidade infantil em áreas urbanas, Río de Janeiro, Fundação JBGE. 
Fundação Sistema Estadual de Ana]ise de Dados [SEADE) (1987). Pesquisa de emprego e desemprego: príncipais resultados, vol. 34s, San Pablo, SEADE.

García, Norberto E. y Víctor E. Tokman (1985). Acumulación, empleo y crisis, Santiago, Chile, PREALC, Investigaciones sobre Empleo (número 25).

Glass, David V. (1938). "Marriage Frequency and Economic Fluctuations in England and Wales, 1851-1934", en L. Hobgen (ed.), Political arithmetic, Londres.

Glewwe, Paul y Dennis De Tray (1988). The Poor During Adjusment: A Case Study of Côte d'Ivoire, Washington, DC., World Bank Living Standards Measurement Study Working Paper, 47.

Goldani, Ana María, Stephen D. McCracken y Thomas W. Pullum (1989). "Demographic Change and Stability in Brazil During a Period of Economic Crisis", ponencia presentada en la reunión anual de la Population Association of America, Baltimore, Md.

Haignere, Clara S. (1983). "The Application of the Free-market Economic Model in Chile and the Effects on the Population's Health Status", en International Journal of Health Services, 13 (3), 389-405.

Hakkert, Ralph (1984). "Conseqüencias da crise nas tendências demográficas de São Paulo", en ABEP, Anais do IVo Encontro Nacional de Estudos de População. Aguas de São Pedro, vol. 3, 1433-1447.

Hogan, Daniel j. (1985). "Demografía e conjuntura, reflexōes metodológicas", Revista Brasileira de Estudos de População, 2 (1), enero/junio, 1-7.

Hojman; David E. [1985]. "Free-market Economic Policies and Infant and Child Mortality in Chile Multiple Regression, Principal Components and Simultaneous Equation Models", University of Liverpool, Centre for Latin American Studies, Occasional Paper, 6.

Hugo, Graeme J. (1984). "The Demographic Impact of Famine a Review", en Graeme J., Hugo y Bruce Currey (eds.), Famine as a Geographical Phenomenon, Riedel, 7-31.

Kane, Penny (1987). "The Demography of Famine", en Genus 43 (1-2), 43-58.

Kirk, Dudley (1960). "The Influence of Business Cycles on Marriage and Birth Rates", en Demographic and Economic Change in Developed Countries, Princeton University Press.

Klein, Emilio y José Wurgaft (1985). La creación de empleo en periodos de crisis, Santiago, Chile, PREALC, Investigaciones sobre Empleo (núm. 24).

Lavell, Allan (1988). "Economic Recession and Urban Labour Market Dynamics in Costa Rica 1978-1984", ponencia presentada en la Conference on the Demography of Inequality in Contemporary Latin America, University of Florida, Gainesville, Flo.

Leser, Walter (1975). "Crescimento da população da cidade de São Paulo entre 1950-1970 e seu reflexo nas condições de saúde pública", en M.C. Andrade et al., Meio ambjente, desenvolvimento $\theta$ subdesenvolvi mento, San Pablo, HUCITEC, 15-44.

Lessa, Ricardo (1987). "Malas prontas", Isto E, 551, 43-46. 
Macedo, Roberto (1984). "Brazilian Children and the Economic Crisis, Evidence from the State of São Paulo", en World Development, 12 (3), 203-221.

MacMahon, B. y S. Yen (1971). "Unrecognized Epidemic of Anencephaly and Spina Bifida", Lancet, 31-33.

Martine, George, Ivany C. Neiva y Marle Macedo (1984). "Migraçāo, crise e outras agruras", en ABEP, Anais do IV Encontro Nacional, San Pablo, vol. 3, 1449-1476.

y Ronaldo García (eds.) [1987). Os impactos sociais de modernização agricola, San Pablo, Caetés.

Meuvret, Jean (1965). "Demographic Crisis in France from the 16th to the 18th Century", en David V. Glass y D.E.C. Eversley (eds.), Population in History Essays in Historical Demography, Londres, Edward Arnold, 507-522.

Ministerio de Trabajo y Servicios Sociales (MTSS) y Dirección General de Estadística y Censos (DGEC) (1977-1986). Encuesta Naciona] de Hogares, Empleo y Desempleo, San José, Costa Rica, MTTs.

Monteiro, Carlos A. (1982). "Contribuição para o estudo do significado da evolução do coeficiente de mortalidade infantil no municipio de São Paulo, SP (Brasil), nas tres últimas décadas (1950-1979)", en Revista de Saúde Pública, 16 (1).

PREALC [1985]. Más allá de la crisis, Santiago, PREALC.

Pressat, Roland [1969). "Interprétation des variations a court terme des taux de la natalité", en United Nations, Annals of the World Population Conference, Belgrado.

Raczynski, Dagmar [1985). "Condiciones socioeconómicas recesivas y morbi-mortalidad infantil", en População e Saúde. Anais do Seminario Latino-Americano, UNICAMP, vol. 2, 303-375.

y César Oyarzo (1981). "¿Por qué cae la tasa de mortalidad infantil en Chile?", Estudios CIEPLAN, 6, 45-84.

Roett, Riordan [1987). "Brazil and the Debt: Will the Cost be too High?" Ponencia presentada en la Conference on the Latin American Debt Problems and Policies, Stanford University, 17-19 de septiembre.

Rosero, Luis (1983). "Social and Economic Policies and their Effects on Mortality: the Costa Rican Case", París, IUSSP-INED.

Sawyer, Donald R. [1989). "The Effects of the Brazilian Economic Crisis on Migration to the Amazon", ponencia presentada en la reunión anual de la Population Association of America, Baltimore, Md.

Secretaria do Planajamento e Coordinaçāo Geral (SEPLAN, Rondônia) (1986). Boletim de Migração, año VI, Porto Velho, SEPLAN.

Sell, Ralph R. y Stephen J. Kunitz (1986). "The Debt Crisis and the End of an Era in Mortality Decline", en Studies in Comparative International Development, 21 (4), 3-30.

Sen, A. (1981). Poverty and Famines: An Essay on Entitlement and Deprivation, Oxford, Clarendon Press.

Silva, Alberto C. da (1986). "Malnutrition in Brazil after 1975", Washington, D.C., World Bank, mimeo.

Singer, Paul I. (1985). "Os efeitos da crise econômica sobre o estado de 
nutrição dos brasileiros", en Marfa C. de S. Minayo y Otavio C. Neto (eds.), A crise e a fome no Brasil e na America Latina, Petrópolis, FASE/Vozes.

Skipp, Victor H. T. (1978). Crisis and Development: En Ecological Case Study of the Forest of Arden, 1570-1674, Nueva York, Cambridge University Press.

Sogner, Solvi (1979). "Nature and Dynamics of Crises (Including Recent Crises in Developing Countries", en Hubert Charbonneau y André Larosse (eds.), The Great Mortalities Methodological Studies of Demographic Crises in the Past, Dolhain, Belgium, Ordina Editions, 311-331.

Solimano, Giorgio y Clara S. Haignere (1983). "Free Market Politics and Nutrition in Chile, a Grim Future After a Short-lived Succoss", Nueva York, Center for Population and Family Health, Columbia University, Documento de trabajo, 7.

Thomas, Brinley (1954). Migration and Economic Growth a Study of Great Britain and the United States, Cambridge, Cambridge University Press.

Trussell, T. James y Anne R. Pebley [1984]. The Potential Impact of Changes in Fertility on Infant, Child, and Maternal Mortality, World Bank Staff Working Paper 698, Washington, D.C.

UNICEF (1987). Ajuste com dimensão humana. Parte II da Edição Completa. Situação Mundial da Infancia, Brasilia, Ideal.

United Nations (1985). Socio-economic Development and Fertility Decline in Costa Rica, United Nations, Departamento of International Economic and Social Affairs, Nueva York.

(1986). Economic recession and specific population groups, United Nations, Nueva York, Departamento of International Economic and Social Affairs.

Van Roy, Ralph (1984). "Undocumented Migration to Venezuela", International Migration Review, 18, 541-557.

Vetter, David M. (1983). "A evolução das condições de saneamento básico da população urbana durante a década de 70 , uma análise preltminar", en Revista Brasileira de Estatistica, 44 (173-174), 181-198.

Villar, Juan Manuel (1984). "Argentine Experience in the Field of Ilegal Inmigration", International Migration Review, 18, 453-473.

Wood, Charles H. (1977). "Infant Mortality Trends and Capitalist Deve]opment in Brazil: the Case of São Paulo and Belo Horizonte"; en Latin American Perspectives, 4 (4), 56-65.

Wood, Charles H. y José A. M. de Carvalho (1988). The Demography of Inequality, Nueva York, Cambridge University Press.

World Bank (1986). Poverty in Latin America, the Impact of Depression, Washington, D.C, World Bank. 